\title{
Relationship Between Left Atrial Appendage Function and Left Atrial Thrombus in Patients With Nonvalvular Chronic Atrial Fibrillation and Atrial Flutter
}

\author{
Tatsuyoshi Narumiya, MD; Tatsuo Sakamaki, MD*; \\ Yuichi Sato, MD; Katsuo Kanmatsuse, MD
}

\begin{abstract}
The relationship between the left atrial appendage (LAA) function, as assessed by transesophageal echocardiography, and the incidence of left atrial thrombus was evaluated in 62 patients with nonvalvular chronic atrial fibrillation (AF; $n=50$ ) and atrial flutter (AFL; $n=12$ ). It was hypothesized that in both AF and AFL not only the LAA flow velocity (LAAFV), but also the frequency of the LAA movement (the LAA flow time, LAAFT) is a major contributing factor to thrombus formation. LAAFT was defined as the average duration of LAA flow with emptying and filling waves. The patients with AF were divided into 2 groups: lone $\mathrm{AF}(\mathrm{n}=14)$ and non-lone $\mathrm{AF}$ $(\mathrm{n}=36)$. LAA thrombus was found in 6 patients with none-lone AF. LAAFV was lower and LAAFT was shorter in patients with thrombus as compared with patients without thrombus $(12.0 \pm 2.2 \mathrm{~cm} / \mathrm{s}$ vs $24.1 \pm 10.6 \mathrm{~cm} / \mathrm{s}$, $68.7 \pm 1.5 \mathrm{~ms}$ vs $72.9 \pm 3.3 \mathrm{~ms}, \mathrm{p}<0.01$, respectively). Patients with AFL had higher LAAFV and longer LAAFT than those with chronic AF. The present data suggest that, in addition to LAAFV, LAAFT characterized LAA function and might serve as a predictor of thrombus formation in chronic AF. With respect to LAA function, patients with lone AF or AFL are at low risk for thrombus formation. (Circ J 2003; 67: 68-72)
\end{abstract}

Key Words: Atrial fibrillation; Atrial flutter; Left atrial appendage function; Left atrial thrombosis; Transesophageal echocardiography

$\mathbf{T}$ he major complications of atrial fibrillation $(\mathrm{AF})$ are hemodynamic compromise and thromboembolism; 6-24\% of all ischemic strokes have been attributed to $\mathrm{AF}^{1,2}$ In these patients, the left atrium (LA), especially the left atrial appendage (LAA), is the presumed site of thrombus formation and a source of arterial thrombus? Transesophageal echocardiography (TEE) is a potentially useful diagnostic imaging modality because it enables reliable assessment of LA thrombus and LAA flow velocity (LAAFV), but the ability of LAAFV alone to predict LA and LAA thrombus in patients with AF remains controversial-8 Although the risk of embolism is thought to be lower with atrial flutter (AFL),-11 recent TEE studies have demonstrated an unexpectedly high incidence of atrial thrombus formation ${ }^{12-15}$ LAA blood drainage is an important factor in the pathogenesis of local flow stasis and thrombus formation, and we hypothesized that in both $\mathrm{AF}$ and $\mathrm{AFL}$ the LAA blood drainage was reduced when the frequency of LAA movement increased. Thus, we examined whether or not LAA flow time (LAAFT), the new index of LAA movement and defined as the average duration of LAA flow with emptying and filling waves, is a useful predictor of thrombus formation.

(Received April 1, 2002; revised manuscript received October 17, 2002; accepted October 23, 2002)

Department of Cardiology, Nihon University Surugadai Hospital, Tokyo and *Department of Internal Medicine, Nihon University School of Dentistry at Matsudo, Chiba, Japan

Mailing address: Tatsuyoshi Narumiya, MD, Department of Cardiology, Nihon University Surugadai Hospital, 1-8-13 Kanda-Surugadai, Chiyoda-ku, Tokyo 101-8309, Japan. E-mail: narunaru@t3.rim.or.jp
The aim of the present study was to evaluate the relationship between LAA function and the incidence of LA thrombus in patients with nonvalvular chronic AF and AFL.

\section{Study Patients \\ From April 1995 to May 2000, we retrospectively} reviewed patients with nonvalvular chronic AF and AFL who had undergone TEE. Patients with left ventricular (LV) systolic dysfunction (LV ejection fraction $<0.5$ ) and patients undergoing anticoagulant therapy were excluded. Nonvalvular chronic AF was defined by conventional electrocardiogram (ECG) on 2 occasions separated by at least 1 month and by the absence of rheumatic heart disease as determined by echocardiography. Lone AF was defined by excluding coronary artery disease (based on clinical or laboratory criteria), hyperthyroidism, valvular heart diseases, congestive heart failure, cardiomyopathy, chronic obstructive pulmonary disease, cardiomegaly on chest X-ray, history of hypertension, age over 60 years, insulin-dependent diabetes, AF only during trauma or surgery, and acute medical illness! ${ }^{16}$

Sixty-two patients (53 male, 9 female; mean age, $60.0 \pm$ 9.7 years) entered the study: 50 had chronic AF and 12 had AFL. The patients with AF were divided into 2 groups: lone $\mathrm{AF}(\mathrm{n}=14)$ and non-lone $\mathrm{AF}(\mathrm{n}=36)$. 


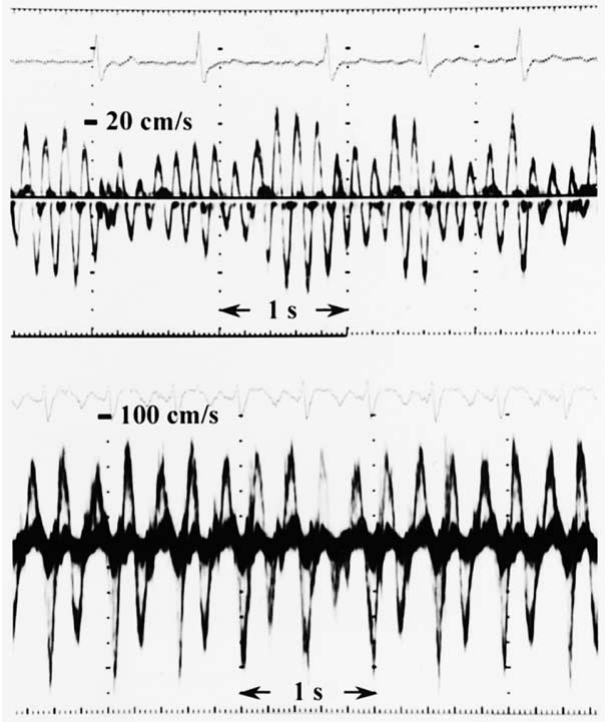

Fig 1. (Top) Pulsed Doppler tracing of the left atrial appendage (LAA) flow pattern in a patient with atrial fibrillation (AF), showing 'saw tooth' flow signals of variable amplitude. (Bottom) Pulsed Doppler tracing of the LAA flow pattern in a patient with atrial flutter (AFL) with a more consistent high velocity, relatively regular, sawtooth flow pattern.



Fig 2. Schema of the left atrial appendage (LAA) flow pattern. Measurements of LAA flow velocity (LAAFV) and LAA flow time (LAAFT).

\section{Transthoracic Echocardiography and Doppler \\ Echocardiography}

Transthoracic echocardiographic studies were performed using a Sonos 500 machine (Hewlett-Packard Co, Andover, MA, USA) with a 2.5- or 3.5-MHz transducer and a Sonos 4500 (Agilent Tech. Co, Andover, MA, USA) with a 2.04.0 MHz transducer. The LA size, LV end-diastolic dimension, and LV ejection fraction were measured from $\mathrm{M}$ mode tracings ${ }^{17}$

\section{Transesophageal Echocardiography}

All TEE studies were performed with the patients in the left lateral decubitus position after they had fasted for more than 5-6h prior to the TEE study to avoid the risk of vomiting and aspiration. After locally anesthetizing the mucosal surface of the hypopharynx by lidocaine, a transesophageal probe $(5-\mathrm{MHz}$ phased array biplane transducer, $\mathrm{n}=59$; or 5MHz multiplane transducer, $n=3$ ) was introduced into the esophagus. The LAA was imaged primarily in 2 basic biplane TEE views; namely, the horizontal short-axis view
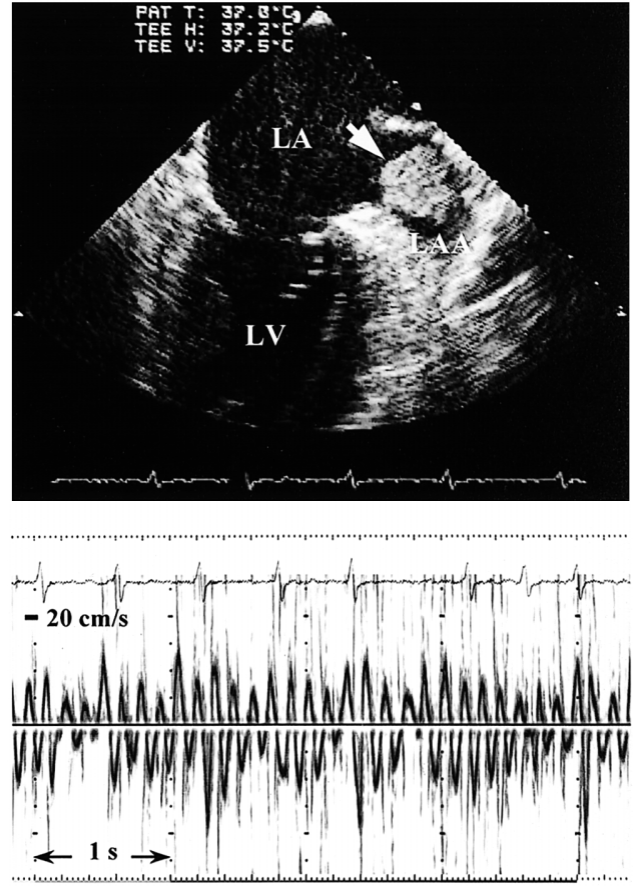

Fig 3. (Top) Transesophageal echocardiographic image of a thrombus (arrow) located in the left atrial appendage (LAA) in a patient with atrial fibrillation. LA, left atrium; LV, left ventricle. (Bottom) LAA flow pattern in the same patient: LAAFV was $12 \mathrm{~cm} / \mathrm{s}$ and LAAFT was $70 \mathrm{~ms}$.

at the base of the heart and a 2-chamber longitudinal view of the LA and LV.

LAAFV was derived while in the longitudinal view by placing the pulsed Doppler sample volume at $1 \mathrm{~cm}$ inside the LAA orifice ${ }^{7,18}$ and recording the Doppler signals for 10 consecutive cardiac cycles on a stripchart at a recording speed of $50 \mathrm{~mm} / \mathrm{s}$ (Fig 1). The LAAFV was measured and averaged from 5 consecutive cardiac cycles. LAAFT was defined as the average duration of LAA flow with emptying and filling waves, and was measured from the average of 3-5 consecutive R-R intervals (Fig 2). The heart rate was determined as the average of 10 consecutive cardiac cycles on ECG.

\section{Left Atrial Thrombus}

The presence of LA or LAA thrombus was defined in the TEE views as (1) masses adhering to the wall of the LA or its appendage, (2) motion independent of the LAA wall, (3) different echogenic density from the LAA wall, and (4) evidence in more than one imaging plane. Particular care was taken to differentiate a LAA thrombus from the pectinate muscle. When discrepancies occurred, the videotape was reviewed until a consensus was reached.

\section{Statistical Analysis}

For each TEE parameter, a threshold analysis using receiver operating characteristic curve was performed to determine the parameter and value that most powerfully discriminated between patients with and without LA thrombus. All the data were expressed as the mean \pm standard deviation. Continuous variables between groups were compared by Mann-Whitney's U test for unpaired observations. A p value less than 0.05 was considered statistically significant. 
Table 1 Patients' Profiles

\begin{tabular}{lcccc}
\hline \hline & AF thrombus $(+)$ & \multicolumn{3}{c}{ Thrombus $(-)$} \\
\cline { 3 - 5 } & $(n=6)$ & $\begin{array}{c}\text { Non-lone } A F \\
(n=30)\end{array}$ & $\begin{array}{c}\text { Lone } A F \\
(n=14)\end{array}$ & $\begin{array}{c}A F L \\
(n=12)\end{array}$ \\
\hline Age (years) & $64.0 \pm 10.0^{*}$ & $61.1 \pm 10.5^{*}$ & $53.4 \pm 5.7$ & $63.1 \pm 8.4^{*}$ \\
Gender (M:F) & $5: 1$ & $23: 7$ & $13: 1$ & $12: 0$ \\
\hline
\end{tabular}

AF, atrial fibrillation; AFL, atrial flutter. ${ }^{*} p<0.05$ vs lone $A F$.

Table 2 Transthoracic Echocardiographic and Hematologic Parameters

\begin{tabular}{lcccc}
\hline \hline & AF thrombus $(+)$ & \multicolumn{3}{c}{ Thrombus $(-)$} \\
\cline { 3 - 5 } & $(n=6)$ & $\begin{array}{c}\text { Non-lone } A F \\
(n=30)\end{array}$ & $\begin{array}{c}\text { Lone } A F \\
(n=14)\end{array}$ & $\begin{array}{c}A F L \\
(n=12)\end{array}$ \\
\hline Heart rate $($ beats/min $)$ & $92.2 \pm 11.4$ & $89.9 \pm 10.3$ & $96.1 \pm 14.5$ & $92.8 \pm 19.2$ \\
LAD $(\mathrm{mm})$ & $48.2 \pm 4.0$ & $44.5 \pm 4.6$ & $40.6 \pm 7.9$ & $38.3 \pm 5.5^{*}$ \\
LVDd $(\mathrm{mm})$ & $51.8 \pm 6.2$ & $49.1 \pm 3.8$ & $48.3 \pm 4.8$ & $50.1 \pm 4.2$ \\
Ejection fraction & $0.60 \pm 0.08$ & $0.64 \pm 0.08$ & $0.60 \pm 0.07$ & $0.66 \pm 0.08$ \\
RBC $\left(\times 10^{4} / \mathrm{\mu l}\right)$ & $497 \pm 68$ & $480 \pm 39$ & $467 \pm 42$ & $459 \pm 43$ \\
Hematocrit $(\%)$ & $44.4 \pm 6.9$ & $45.4 \pm 3.7$ & $45.7 \pm 2.9$ & $42.5 \pm 4.7$ \\
Platelet $\left(\times 10^{4}\right.$ pl $\left.l\right)$ & $24.9 \pm 14.9$ & $20.5 \pm 6.3$ & $18.6 \pm 4.9$ & $20.8 \pm 4.2$ \\
\hline
\end{tabular}

$L A D$, left atrial dimension; $L V D d$, left ventricular end-diastolic dimension; $R B C$, red blood cell count. * $p<0.05$ vs Thrombus (+).

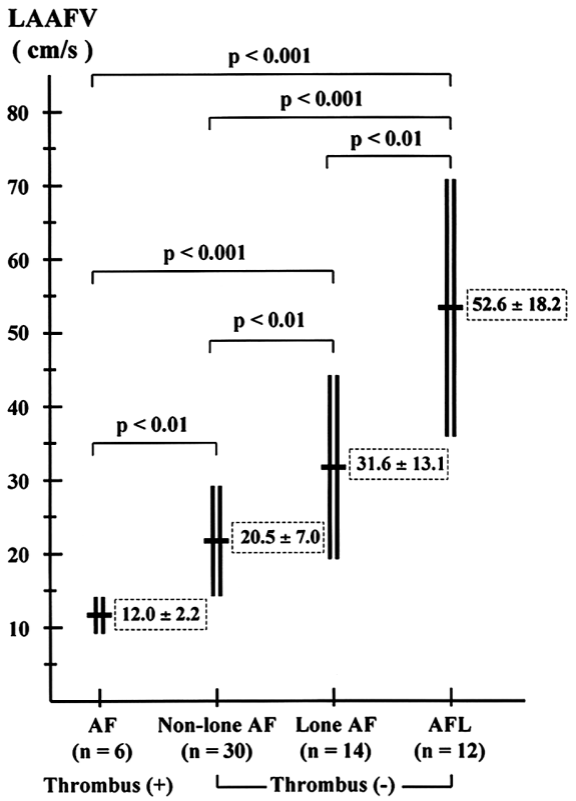

Fig 4. Left atrial appendage flow velocity (LAAFV) in patients with thrombus, non-lone AF, lone AF, and AFL.

\section{Results}

\section{Left Atrial Thrombus}

TEE demonstrated LAA thrombus in 6 of 62 patients (Fig 3), and of these patients, 5 had hypertension, 1 had ischemic heart disease, 1 had diabetes mellitus and clinical episodes of cerebral infarction, and 1 had clinical episodes of systemic embolism involving the kidneys and spleen. The remaining 1 of the 6 had echocardiographic evidence of hypertrophic non-obstructive cardiomyopathy and history of cerebral infarction. Thrombus was not found in patients with lone AF or AFL (Table 1).

Transthoracic Echocardiographic Parameters

In patients with $\mathrm{AF}$, the LA dimension was not signifi-

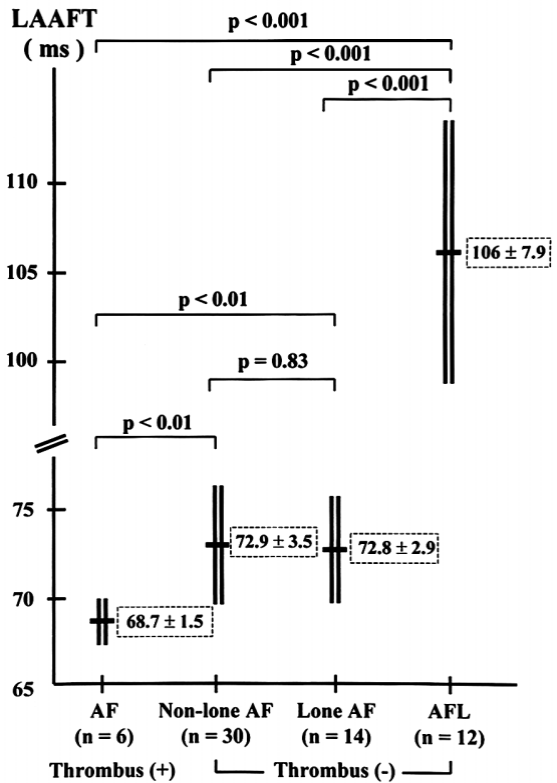

Fig 5. Left atrial appendage flow time (LAAFT) in patients with thrombus, non-lone AF, lone AF, and AFL.

cantly different between patients with and without thrombus $(48.2 \pm 4.0 \mathrm{~mm}$ vs $43.2 \pm 6.0 \mathrm{~mm}, \mathrm{p}=0.14)$. However, the LA dimension in patients with AFL was significantly smaller than that in patients with chronic AF. There were no significant differences in the other parameters among patients with LA thrombus or without thrombus or with AFL (Table 2). In addition, the heart rate did not differ significantly among patients with any type of chronic AF or with AFL.

\section{Hematologic Parameters}

There were no significant differences in the red blood cell count, hematocrit and platelet count among patients with any type of chronic AF or with AFL (Table 2). 


\section{Parameters of LAA Function}

In patients with $\mathrm{AF}, \mathrm{LAAFV}$ was lower in patients with LAA thrombus compared with those without thrombus $(12.0 \pm 2.2 \mathrm{~cm} / \mathrm{s}$ vs $24.1 \pm 10.6 \mathrm{~cm} / \mathrm{s}, \mathrm{p}<0.01)$. Of the 44 chronic AF patients without thrombus, LAAFV was higher in patients with lone $\mathrm{AF}$ than those with non-lone $\mathrm{AF}$ $(31.6 \pm 13.1 \mathrm{~cm} / \mathrm{s}$ vs $20.5 \pm 7.0 \mathrm{~cm} / \mathrm{s}, \mathrm{p}<0.01)$. LAAFV in patients with AFL was higher than in any group with chronic AF (Fig 4). LAAFT was shorter in patients with thrombus than those without thrombus $(68.7 \pm 1.5 \mathrm{~ms}$ vs $72.9 \pm 3.3 \mathrm{~ms}$, $\mathrm{p}<0.01)$. However, patients with lone or non-lone AF without thrombus did not differ with respect to LAAFT $(72.8 \pm 2.9 \mathrm{~ms}$ vs $72.9 \pm 3.5 \mathrm{~ms}, \mathrm{p}=0.83)$. LAAFT was significantly longer in patients with AFL compared with patients with chronic AF (Fig 5).

Statistical analysis using receiver operating characteristic curve identified the 2 TEE predictors of LA thrombus: (1) LAAFV $<15 \mathrm{~cm} / \mathrm{s}$ and (2) LAAFT $<71 \mathrm{~ms}$ (Fig 6). The sensitivity, specificity and accuracy for predicting the patients with thrombus were $100 \%, 79.6 \%$ and $82.0 \%$ when $\mathrm{LAAFV}=15 \mathrm{~cm} / \mathrm{s}$ was used as the cut-off point and $100 \%, 79.6 \%$ and $82.0 \%$ when LAAFT $=71 \mathrm{~ms}$ was used. When $\mathrm{LAAFV}=15 \mathrm{~cm} / \mathrm{s}$ and $\mathrm{LAAFT}=71 \mathrm{~ms}$ were combined, the sensitivity, specificity and accuracy was $100 \%$, $90.9 \%$ and $92.0 \%$, respectively.

\section{Discussion}

In $\mathrm{AF}$, the clinical significance of LAAFV as a predictor of LA thrombus has been established,8,19-21 but its accuracy has been questioned; for example, Mügge et al did not clearly establish the relationship between LAAFV and the incidence of thrombus? We hypothesized that not only LAAFV, but also the frequency of the LAA movement as determined by the duration of LAA flow with emptying and filling waves is a major contributing factor to thrombus formation. Therefore, we investigated whether or not LAAFT was a useful index for predicting LA thrombus and whether the combination of LAAFT and LAAFV might be a superior predictor of thrombus over LAAFV alone.

In fact, the present study demonstrated that LAAFT is a powerful predictor of LA thrombus, at least equivalent to LAAFV. The threshold value of LAAFT $=71 \mathrm{~ms}$ clearly separated subsets of patients with and without thrombus, with a sensitivity of $100 \%$, specificity $79.5 \%$ and accuracy $82.0 \%$. The combination of threshold values of LAAFV = $15 \mathrm{~cm} / \mathrm{s}$ and LAAFT $=71 \mathrm{~ms}$ further discriminated patients with thrombus from those without thrombus, with a sensitivity of $100 \%$, specificity $90.9 \%$ and accuracy $92.0 \%$.

The second major finding is that both LAAFV and LAAFT clearly differentiated subsets of patients with AFL from those with chronic AF (Figs 4,5). Patients with AFL have traditionally been considered at low risk for thromboembolism because of the presence of organized mechanical atrial activity9-11 However, recent TEE studies frequently reported LA thrombus in patients with AFL;12-14 Black et al found the incidence of thrombus in patients with AFL equivalent to those with chronic $\mathrm{AF}$ (14\% vs $12 \%) !^{2}$ In other studies of patients with AFL, $5(11 \%)$ of $47^{13}$ and 5 (21\%) of $24^{14}$ were found to have LA thrombus. In the present study, however, the association of AFL and LA thrombus was not observed and the discrepancy in the data may stem from the differences in patient selection. The other studies ${ }^{12-14}$ included patients with decreased LV systolic function, whereas the present study had only

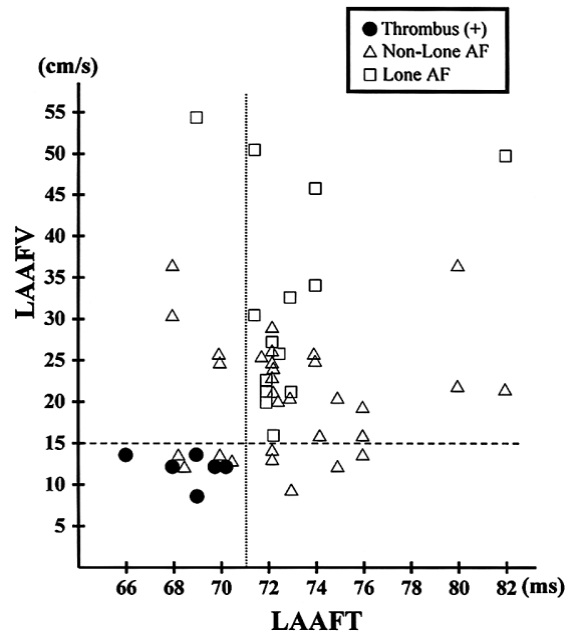

Fig 6. Left atrial appendage flow velocity (LAAFV) and left atrial appendage flow time (LAAFT) in patients with AF. Threshold values of $\mathrm{LAAFV}=15 \mathrm{~cm} / \mathrm{s}$ and LAAFT $=71 \mathrm{~ms}$ clearly discriminate patients with thrombus from those without thrombus.

patients with normal LV ejection fraction. Santiago et al ${ }^{11}$ reported that AFL was associated with a significantly higher LAAFV compared with patients with chronic AF $(42 \pm 18 \mathrm{~cm} / \mathrm{s}$ vs $17 \pm 10 \mathrm{~cm} / \mathrm{s}, \mathrm{p}<0.001)$, and we also documented higher LAAFV in patients with AFL when compared with those with chronic AF. In addition, the present data showed a longer LAAFT and a relatively regular LAA flow pattern in patients with AFL compared with those with AF (Fig 1). Thus, it is reasonable to speculate that high LAAFV and long LAAFT play a role in inhibiting thrombus formation in patients with AFL.

Whether LAAFV is a reliable predictor of LA thrombus has been investigated in several studies. Archer et al ${ }^{5}$ showed that patients with LA thrombus tended to have lower LAAFV than those without thrombus, although this

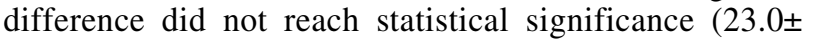
$6.4 \mathrm{~cm} / \mathrm{s}$ vs $34.0 \pm 14.8 \mathrm{~cm} / \mathrm{s}, \mathrm{p}=0.11$ ). Furthermore, Heppell et $\mathrm{al}^{6}$ and Pozzoli et $\mathrm{al}^{8}$ found a significant correlation between LAAFV and the incidence of LA thrombus. However, Mügge et $\mathrm{al}^{7}$ divided their cohort into patients with 'high flow profile' (LAAFV $\geq 25 \mathrm{~cm} / \mathrm{s}$ ) and 'low flow profile' (LAAFV $<25 \mathrm{~cm} / \mathrm{s}$ ) and did not clearly establish a relationship between LAAFV and thrombus. The difference between that study ${ }^{7}$ and the present one is that Mügge et al included patients with paroxysmal $\mathrm{AF}$ and acute $\mathrm{AF}$ in their patient population whereas we included only patients with chronic AF. In addition, we excluded patients undergoing anticoagulation therapy, and therefore, the interference of anticoagulation therapy on thrombus formation ${ }^{21}$ was avoided.

\section{Study Limitation}

The major limitation of this study is the small patient population. We intentionally excluded patients with LV systolic dysfunction ${ }^{12,14,22,23}$ and those undergoing anticoagulation therapy, ${ }^{21}$ because inclusion of the former might have led to overestimation of the incidence of thrombus and the latter to underestimation. However, further study with a larger patient population with prospective data analysis is obviously required to establish the relationship between LV systolic function and the incidence of throm- 
bus, and to evaluate the efficacy of anticoagulation therapy on thrombus formation.

In the present study, we used both biplane and multiplane transducers with the same observers. Although no previous data are available concerning the difference in thrombus detectability by the same observers, the ability to evaluate LAA function is comparable between the 2 methods?,13,22,24 We were also concerned that various antiarrhythmic agents may affect LAA function. Bilge et $\mathrm{al}^{25}$ showed that $\beta$-blocking agents may further deteriorate LAA function in patients with chronic AF. On the other hand, verapamil may increase LAA function? ${ }^{24}$ Twelve of the present patients were taking these medications, but discontinuation was considered to be unethical. Further prospective study is obviously needed to clarify the effect of these agents on LAAFV and LAAFT.

In conclusion, the present results suggest that, in addition to LAAFV, LAAFT characterizes LAA function and might serve as a predictor of thrombus formation in nonvalvular chronic AF. The data also suggest that combining these 2 indices is useful for predicting LAA thrombus. Further analysis with a larger patient population and prospective data analysis is needed to establish the relationship of these indices and the incidence of LA thrombus.

\section{References}

1. Cardiogenic brain embolism. The second report of the Cerebral Embolism Task Force. Arch Neurol 1989; 46: 727-743.

2. Miyazaki S, Ito T, Suwa M, Nakamura T, Kobashi A, Kitaura Y. Role of transesophageal echocardiography in the prediction of thromboembolism in patients with chronic nonvalvular atrial fibrillation. Jpn Circ J 2001; 65: 874-878.

3. Abe Y, Asakura T, Gotou J, Iwai M, Watanabe Y, Sando M, et al. Prediction of embolism in atrial fibrillation: Classification of left atrial thrombi by transesophageal echocardiography. Jpn Circ J 2000; 64: $411-415$.

4. Fatkin D, Scalia G, Jacobs N, Burstow D, Leung D, Walsh W, et al. Accuracy of biplane transesophageal echocardiography in detecting left atrial thrombi. Am J Cardiol 1996; 77: 321-323.

5. Archer SL, James KE, Kvernen LR, Cohen IS, Ezekowitz MD, Gronick CC. Role of transesophageal echocardiography in the detection of left atrial thrombus in patients with chronic nonrheumatic atrial fibrillation. Am Heart J 1995; 130: 287-295.

6. Heppell RM, Berkin KE, McLenachan JM, Davies JA. Haemostatic and haemodynamic abnormalities associated with left atrial thrombosis in non-rheumatic atrial fibrillation. Heart 1997; 77: 407-411.

7. Mügge A, Kuhn H, Nikutta P, Grote J, Lopez JA, Daniel WG. Assessment of left atrial appendage function by biplane transesophageal echocardiography in patients with nonrheumatic atrial fibrillation: Identification of a subgroup of patients at increased embolic risk. J Am Coll Cardiol 1994; 23: 599-607.

8. Pozzoli M, Febo O, Torbicki A, Tramarin R, Calsamiglia G, Cobelli F, et al. Left atrial appendage dysfunction: A cause of thrombosis? Evidence by transesophageal echocardiography-Doppler studies. $J$ Am Soc Echocardiogr 1991; 4: 435-441.
9. Arnold AZ, Mick MJ, Mazurek RP, Loop FD, Trohman RG. Role of prophylactic anticoagulation for direct current cardioversion in patients with atrial fibrillation or atrial flutter. J Am Coll Cardiol 1992; 19: $851-855$.

10. Roy D, Marchand E, Gagne P, Chabot M, Cartier R. Usefulness of anticoagulant therapy in the prevention of embolic complications of atrial fibrillation. Am Heart J 1986; 112: 1039-1043.

11. Santiago D, Warshofsky M, Li Manderi G, Di Tullio M, Coromilas J, Reiffel J, et al. Left atrial appendage function and thrombus formation in atrial fibrillation-flutter: A transesophageal echocardiographic study. J Am Coll Cardiol 1994; 24: 159-164.

12. Black IW, Hopkins AP, Lee LC, Walsh WF. Evaluation of transesophageal echocardiography before cardioversion of atrial fibrillation and flutter in nonanticoagulated patients. Am Heart J 1993; 126: $375-381$.

13. Irani WN, Grayburn PA, Afridi I. Prevalence of thrombus, spontaneous echo contrast, and atrial stunning in patients undergoing cardioversion of atrial flutter: A prospective study using transesophageal echocardiography. Circulation 1997; 95: 962-966.

14. Bikkina M, Alpert MA, Mulekar M, Shakoor A, Massey CV, Covin FA. Prevalence of intraatrial thrombus in patients with atrial flutter. Am J Cardiol 1995; 76: 186-189.

15. Thumala A, Parra C, Maragano P, Puelma A, Florenzano F. Thromboembolic risk factors in atrial flutter: Transesophageal echocardiographic study. Rev Med Chil 2000; 128: 1327-1334.

16. Kopecky SL, Gersh BJ, McGoon MD, Whisnant JP, Holmes DR Jr, Ilstrup DM, et al. The natural history of lone atrial fibrillation; A population-based study over three decades. N Engl J Med 1987; 317: 669-674.

17. Sahn DJ, DeMaria A, Kisslo J, Weyman A. Recommendations regarding quantitation in M-mode echocardiography: Results of a survey of echocardiographic measurements. Circulation 1978; 58: $1072-1083$.

18. Chan SK, Kannam JP, Douglas PS, Manning WJ. Multiplane transesophageal echocardiographic assessment of left atrial appendage anatomy and function. Am J Cardiol 1995; 76: 528-530.

19. Zabalgoitia M, Halperin JL, Pearce LA, Blackshear JL, Asinger RW, Hart RG. Transesophageal echocardiographic correlates of clinical risk of thromboembolism in nonvalvular atrial fibrillation: Stroke Prevention in Atrial Fibrillation III Investigators. J Am Coll Cardiol 1998; 31: $1622-1626$.

20. Pollick C, Taylor D. Assessment of left atrial appendage function by transesophageal echocardiography: Implications for the development of thrombus. Circulation 1991; 84: 223-231.

21. Kimura M, Wasaki Y, Ogawa H, Nakatsuka M, Wakeyama T, Iwami $\mathrm{T}$, et al. Effect of low-intensity warfarin therapy on left atrial thrombus resolution in patients with nonvalvular atrial fibrillation: A transesophageal echocardiographic study. Jpn Circ J 2001; 65: 271 - 274.

22. Tsai LM, Lin LJ, Teng JK, Chen JH. Prevalence and clinical significance of left atrial thrombus in nonrheumatic atrial fibrillation. Int $J$ Cardiol 1997; 58: 163-169.

23. Cemri M, Timurkaynak T, Ozdemir M, Boyaci B, Yalcin R, Cengel A, et al. Effects of left ventricular systolic dysfunction on left atrial appendage and left atrial functions in patients with chronic nonvalvular atrial fibrillation. Acta Cardiol 2002; 57: 101-105.

24. Daoud EG, Marcovitz P, Knight BP, Goyal R, Man KC, Strickberger SA, et al. Short-term effect of atrial fibrillation on atrial contractile function in humans. Circulation 1999; 99: 3024-3027.

25. Bilge M, Guler N, Eryonucu B, Erkoc R. Does acute-phase beta blockade reduce left atrial appendage function in patients with chronic nonvalvular atrial fibrillation? J Am Soc Echocardiogr 2001; 14: $194-199$. 